\title{
BMJ Open Effect of tranexamic acid on the prognosis of patients with traumatic brain injury undergoing craniotomy: study protocol for a randomised controlled trial
}

Bei Wu, Yu Lu (D) , Yun Yu, Hongli Yue, Jie Wang, Yingzi Chong, Weihua Cui (D)

To cite: Wu B, Lu Y, Yu Y, et al. Effect of tranexamic acid on the prognosis of patients with traumatic brain injury undergoing craniotomy: study protocol for a randomised controlled trial. BMJ Open 2021;11:e049839. doi:10.1136/ bmjopen-2021-049839

- Prepublication history for this paper is available online. To view these files, please visit the journal online (http://dx.doi org/10.1136/bmjopen-2021049839).

YL and WC contributed equally.

Received 03 February 2021 Accepted 21 October 2021
Check for updates

(c) Author(s) (or their employer(s)) 2021. Re-use permitted under CC BY-NC. No commercial re-use. See rights and permissions. Published by BMJ.

Department of Anaesthesiology, Beijing Tian Tan hospital, Capital Medical University, Beijing, China

\section{Correspondence to}

Dr Yu Lu;

luyutiantan@163.com and

Dr Weihua Cui;

weihuacui@ccmu.edu.cn

\section{ABSTRACT}

Introduction Abnormal coagulation function aggravates the prognosis of patients with traumatic brain injury (TBI). It was reported that the antifibrinolytic drug tranexamic acid (TXA) could reduce intracranial haemorrhage and mortality in non-operative patients with TBI. However, there is a lack of evaluation of TXA in patients with TBI undergoing craniotomy.

Methods and analysis This is a single-centre randomised controlled, double-blind, parallel study aiming to investigate the effectiveness and safety of TXA in patients with TBI during the perioperative period. Blood loss and transfusion, neurological function, adverse events, mortality and serum immune-inflammatory cytokines will be collected and analysed.

Ethics and dissemination Ethical approval has been granted by the Medical Ethics Committee of Beijing Tian Tan Hospital, Capital Medical University (reference number KY 2020-136-03). The results of this study will be disseminated through presentations at scientific conferences and publication in peer-reviewed journals. Trial registration number ChiCTR2100041911.

\section{INTRODUCTION}

About 1.5 million people die each year from traumatic brain injury (TBI), which has become the leading cause of death and disability worldwide. Brain tissue injury and haemorrhage are the main clinical manifestations of TBI. Systemic inflammatory reaction and abnormal coagulation function after TBI can cause secondary brain injury and aggravate cerebral haemorrhage, which has an extremely adverse effect on the prognosis.

In recent years, many studies ${ }^{1-3}$ have shown that abnormal coagulation function is common in patients with TBI and is one of the main causes of secondary brain injury. The incidence of coagulation dysfunction on admission is about $26 \%$ in patients with TBI, and over $60 \%$ of them have severe TBI. ${ }^{4}$ A prospective study found that patients with
Strengths and limitations of this study

- This is a randomised controlled, double-blind, parallel study to test the hypothesis that tranexamic acid could improve prognosis of patients with traumatic brain injury (TBI) undergoing craniotomy.

- The study will provide evidence for optimising perioperative treatment of patients with TBI undergoing craniotomy.

- There are nearly 500 cases of patients with TBI that need neurosurgery every year at Beijing Tian Tan Hospital, Capital Medical University. It is feasible and reasonable to complete the trial in 3 years.

- The study may not be generalised to other populations since it is a single-centre trial.

TBI with abnormal coagulation function had a 4.7-fold increase in mortality and an increased risk of poor prognosis compared with those without abnormal coagulation function. ${ }^{5}$ Some studies ${ }^{2-4} 6$ have confirmed the interaction between abnormal coagulation function, hyperfibrinolysis and systemic inflammatory reactions.

Tranexamic acid (TXA) has antifibrinolytic and anti-inflammatory effects. Studies have reported that early use of TXA can reduce blood loss and mortality in patients with TBI with non-surgical treatment. ${ }^{78}$ Some studies $^{6-12}$ have suggested that the use of TXA could reduce the mortality and disability of patients with TBI and probably does not increase the risk of adverse events (AEs). TXA may be a potential treatment for patients with TBI.

It is generally believed that the abnormal coagulation function after TBI is closely related to the degree of brain tissue damage. de Oliveira Manoel et al have shown that more than $60 \%$ of patients with severe TBI are accompanied by abnormal coagulation 
function. ${ }^{4}$ The incidence of abnormal coagulation function is increased in patients with TBI combined with systemic injury compared with patients with simple TBI. ${ }^{13}$

Kearney et al have found that patients with severe TBI have a higher mortality rate and a higher degree of abnormality in Glasgow Coma Scale (GCS), Simplified Acute Physiological Score and disseminated intravascular coagulation. ${ }^{14}$ Urgent decompressive craniotomy is recommended for patients with severe TBI with severe craniocerebral trauma, severe cerebral edema, epidural haematomas and subdural haematomas to reduce intracranial pressure (ICP) and mortality and to improve neurological clinical outcomes. ${ }^{15} 16$ For patients with severe TBI complicated by acute traumatic coagulopathy and severe systemic inflammatory response, prompt correction of coagulopathies and anti-inflammatory treatment should be taken into consideration. ${ }^{16}{ }^{17}$ It is not clear whether the use of TXA can improve the prognosis of patients with TBI requiring emergency craniotomy. Further research is still needed to determine the effect of TXA on the systemic inflammatory reaction and coagulation function in patients with TBI undergoing craniotomy procedures.

To sum up, this study intends to evaluate the efficacy and safety of intravenous administration of TXA in patients with TBI undergoing craniotomy procedures. We also aimed to explore the impact of TXA on systemic inflammatory response, coagulation function, the relationship between these effects and prognosis. This study will provide the basis for further development of perioperative TXA usage and optimise perioperative treatment plan for patients with TBI.

\section{Hypothesis to be tested}

In patients with TBI undergoing craniotomy, intravenous administration of TXA improves the patients' clinical outcome and alleviates systemic inflammatory response and coagulation dysfunction.

\section{SPECIFIC OBJECTIVES \\ Primary objectives}

The primary objective of this study was to measure whether intravenous administration of TXA improves the Glasgow Outcome Scale-Extended (GOSE) outcome in patients with TBI undergoing craniotomy procedures at discharge.

\section{Secondary objectives}

The secondary objectives of this study include the following aspects:

1. Bleeding: measure whether intravenous administration of TXA for patients with TBI undergoing craniotomy procedures could reduce intraoperative blood loss and blood transfusion.

2. Safety: observe whether intravenous administration of TXA could increase thrombotic events and postoper- ative infections for patients with TBI undergoing craniotomy.

3. Clinical outcome: investigate whether perioperative administration of TXA could improve the Coma Recovery Scale-Revised (CRS-R) at discharge and reduce the in-hospital and 30-day mortality rates of patients with TBI undergoing craniotomy.

\section{Exploratory objectives}

We will do explored laboratory tests to examine whether TXA could reduce the level of plasma traumatic stress factors, inflammation biomarkers and the abnormalities of coagulation indicators.

\section{METHODS AND ANALYSIS}

\section{Study design and setting}

This study is a single-centre, randomised, double-blind placebo-controlled parallel study to explore the effect of intravenous administration of TXA on the clinical outcome of patients with TBI undergoing craniotomy. From February 2021 to November 2023, patients with TBI undergoing emergency craniotomy will be recruited from Beijing Tian Tan Hospital, Capital Medical University. Data will be collected consecutively from patients after written informed consent is obtained.

\section{Inclusion (eligibility) criteria}

1. Patients aged 18-65 years with TBI scheduled for a craniotomy haematoma removal surgery.

2. Availability of informed consent.

\section{Exclusion criteria}

1. History of allergy to TXA.

2. Taking thrombin.

3. Taking anticoagulants.

4. History of chronic coagulation disorder.

5 . History of thrombosis (deep vein thrombosis, pulmonary embolism, cerebral thrombosis, myocardial infarction and thrombophlebitis).

6. History of renal insufficiency.

7. Pregnancy.

8. History of epilepsy.

9. With mental illness.

\section{Intervention}

The TXA group will be given TXA $2 \mathrm{~g}(40 \mathrm{~mL})$. After the patient is routinely monitored in the operating room and the peripheral vein is established, injections will be pumped at the rate of $80 \mathrm{~mL} /$ hour for $15 \mathrm{~min}$ (loading dose $20 \mathrm{~mL}, 1 \mathrm{~g}$ ) and then $2.5 \mathrm{~mL} /$ hour until the injection is completed (maintenance dose $20 \mathrm{~mL}, 1 \mathrm{~g}$, for 8 hours).

\section{Placebo}

The placebo group will be receiving an equal volume of normal saline delivered at the same rate as the TXA group.

\section{Recruitment procedure and informed consent}

Throughout the study period, a professional full-time quality control staff is in charge of contacting with the 
emergency department. When patients with TBI arrive at the hospital, this staff will screen the patients for eligibility. All participants in this trial will sign informed consent documents. A detailed information regarding the study focusing on the duration, collection of blood samples and the follow-up plan will be provided. The consent forms will be filled up by the anaesthesiologist who is in charge of the anaesthesia. A copy of the consent form along with the information sheets will be provided to each participant. In the consent form, there is a separate provision for consent of biological samples where the participants may agree or refuse. Patients with TBI undergoing neurosurgery are always unconsciousness. Therefore, the entrusted legal representative of the participants has the right to obtain all relevant information and to sign the consent. They will be given sufficient time to decide whether to participate in this study. In case of illiterate representatives, we will obtain thumbprints after getting signature from an impartial witness who is not part of the research team. They will be allowed to withdraw their consent or to discontinue participation without restrictions at any time during the study. The confidentiality of participant records will be protected.

\section{Allocation and randomisation}

After meeting the eligibility criteria and signing the informed consent to participate in the study, 1288 patients will be randomly allocated in a 1:1 ratio into one of two groups: (1) TXA group and (2) placebo group. Each patient is assigned a packet according to his/her ID number, which is sequentially numbered. This packet is labelled with only general study information and the unique ID number. The list that links the ID number to the randomisation code is kept with the statistician who generates it using Stata software V.15.1. The statistician is otherwise not involved in the study.

\section{Blinding}

After the informed consent form is signed and the patient enters the operating room, the full-time quality control staff not involved in clinical treatment will prepare TXA/ placebo syringes according to the randomisation code for the patients.

Patients/representatives, anaesthesiologists and investigators who are responsible for enrolling and observing the primary and secondary outcomes will be concealed throughout the study. Blinding will be discontinued after all data collection is completed. Furthermore, serious life-threatening AEs leading to prolonged hospital stay or death will be reported to the principal investigator (PI), and the blinding will be broken following consultation with the PI if necessary.

\section{Timeline}

The trial was registered at chictr.org.cn on 10 January 2021 (identifier ChiCTR2100041911). The study was approved by the institutional review board at Beijing Tian Tan Hospital, Capital Medical University on 5 January
2021 (reference number KY2020-136-03). The first participant in the study was enrolled in February 2021 and we plan to enroll for 3 years. End of follow-up of patients will be completed approximately 30 days after the last enrolment.

\section{Anaesthesia management}

Standardised procedures will be applied. ${ }^{15} 1819$ After the patient enters the operating room (room temperature is $24^{\circ} \mathrm{C} \sim 26^{\circ} \mathrm{C}$ ), standard routine monitoring will be applied, including non-invasive blood pressure, electrocardiography, pulse oxygen saturation, end-tidal carbon dioxide partial pressure, and body temperature. The experimental medicines will be administrated intravenously. Induction drugs will include sufentanil $(0.3 \sim 0.4 \mathrm{mg} / \mathrm{kg})$, etomidate $(0.3 \sim 0.4 \mathrm{mg} / \mathrm{kg})$ and rocuronium $(0.6 \mathrm{mg} / \mathrm{kg})$. After induction, endotracheal intubation will follow, and the respiratory parameters will be adjusted to maintain $\mathrm{P}_{\mathrm{ET}} \mathrm{CO}_{2}$ within physiological range $\left(35 \sim 45 \mathrm{cmH}_{2} \mathrm{O}\right)$. Arterial and central venous catheterisation will be established. Intraoperative anaesthesia will be adjusted with propofol (2 6 $\mathrm{mg} / \mathrm{kg} /$ hour), remifentanil $(0.1 \sim 0.2 \mathrm{mg} / \mathrm{kg} / \mathrm{min})$ and sevoflurane $(0.5 \sim 1.0 \%)$ to maintain the mean arterial pressure $\geq 60 \mathrm{mmHg}$, and muscle relaxants will be added when necessary. After the operation, the tracheal catheter will be kept or removed (according to circumstances of patients) and patients will be transferred to the intensive care unit (ICU) or neurosurgical ward.

\section{Laboratory procedures}

Blood (3 mL) will be collected from the patients before anaesthesia, 1 and 7 days after surgery, and will be centrifuged at 3000 rotations for $10 \mathrm{~min}$; the supernatant will be collected and stored at $-80^{\circ} \mathrm{C}$.

The blood sample will be analysed for the following:

1. Traumatic stress factors: adcorticotropic hormone $(\mathrm{ACTH})$, epinephrine (AD), norepinephrine (NE), cortisol and lactic acid.

2. Immune inflammatory factors: human monocyte chemotactic protein-1 (McP-1), tumour necrosis factor alpha (TNF- $\alpha), \mathrm{C}$ reactive protein (CRP), peripheral blood leucocyte count and classification, interleukin (IL)-1, IL-6 and IL-10.

\section{Follow-up}

Clinical prognosis of postoperative outcomes will be collected through telephone or interview 30 days after admission date. These will include neurological recovery, reoperation, major postoperative complications, death, etc.

\section{OUTCOMES}

\section{Primary outcome}

The primary outcome is the $\operatorname{GOSE}^{20}$ at discharge. The GOSE is the most highly cited outcome scale in studies of TBI. In a review of outcome measures, Nichol and colleagues listed five features of the ideal outcome scale 
for head injury: it should be logistically simple, reliable, valid, stable and free to administer. ${ }^{21}$ The GOSE meets all of these criteria and is widely recommended as the main outcome measure in studies of TBI. ${ }^{22-24}$ GOSE includes eight categories: (1) dead, (2) vegetative state, (3) lower severe disability, (4) upper severe disability, (5) lower moderate disability, (6) upper moderate disability, (7) lower good recovery and (8) upper good recovery. GOSE $1 \sim 4$ are defined as unfavourable outcomes, GOSE $5 \sim 8$ are defined as favourable outcomes.

At the day of the discharge, the specially trained staff will go to the ward or ICU to do the evaluation of GOSE. The discharge time will be determined by the neurosurgeon. A patient with TBI will be allowed to discharge when meeting the following conditions: (1) intracranial haematoma is in stable absorption phase; (2) ICP is stable, no more dehydration treatment is needed; (3) no more surgical treatment is needed; (4) severe complications are controlled well; and (5) incision is dry without infection and effusion.

\section{Secondary outcome}

1. Intraoperative blood loss: the intraoperative blood loss will be calculated based on the previous study. ${ }^{25}$ The blood loss via the suction will be determined by subtracting the added fluids (heparin and saline solutions) from the total volume contained in the surgical canister. The cotton slivers and pieces used during operation will also be calculated at the end of the surgery. A soaked sliver equals $5 \mathrm{~mL}$ of blood. A soaked piece equals $1 \mathrm{~mL}$ of blood.

2. Blood transfusion: we will record the blood transfusion volume during operation and postoperatively in the ICU and ward, including red blood cells, fresh frozen plasma, fibrinogen (Fbg) and platelets.

3. Thrombotic events: we will observe the incidence of thrombotic events during hospitalisation, including myocardial infarction, pulmonary embolism, deep vein thrombosis and cerebral infarction.

4. Infections: we will record the incidence of postoperative infection complications, including pneumonia, incision infection, intracranial infection and urinary system infection.

5. CRS-R ${ }^{26}$ : the specially trained staff will estimate the CRS-R of all the participants at the day of discharge. We will choose a cut-off score of 8 in our assessment because of the best odds avoiding false-positive and false-negative errors in our analysis. ${ }^{27}$

6. Thirty-day mortality rate after surgery.

7. In-hospital mortality rate.

\section{Exploratory outcomes}

1. Hospitalisation length and costs: we will record the days of hospitalisation after surgery. This will be calculated from the second day after surgery to the discharge day. The hospitalisation costs include the total cost, drug costs, examination and laboratory costs and nursing costs. These will be analysed separately.
2. Traumatic stress and systemic inflammatory response: to evaluate the effect of TXA on traumatic stress and systemic inflammatory response, we will examine the following plasma biomarkers: ACTH, AD, NE, cortisol, lactic acid, McP-1, TNF- $\alpha$, CRP, peripheral blood leucocyte count and classification, IL-1, IL-6 and IL-10.

3. Coagulation functions: the coagulation function and thromboelastogram will be examined before surgery, 1 and 7 days after surgery. The coagulation function test includes the following indicators: thrombin time, Fbg, activated partial thromboplastin time, international standardised ratio, prothrombin time and fibrin degradation products.

\section{Safety considerations, safety monitoring and AE reporting}

All AEs and severe adverse events (SAEs) will be recorded by the study staff. All immediate AE interventions will be documented for all participants. All AEs and SAEs will be followed up until resolution or stabilisation as judged by anaesthesiologist or neurosurgeon and the PI. All SAEs will be followed up until satisfactory resolution or until the treating physicians and the PI deem the event to be chronic or the participant to be stable.

SAEs, which include critical or life-threatening complications or death, will be documented from the time of enrolment throughout the study period. All SAEs and AEs will be reported to the ethics committees and the data and safety monitoring board (DSMB) of the study within 24 hours of awareness of the event.

\section{Data and safety monitoring boards}

The DSMB comprises an anaesthesiologist, a neurosurgeon and a statistician. The DSMB is independent from the sponsor and has no competing interest. The DSMB members will decide the study stopping rules and review the AEs and SAEs reported in the study. They will determine the severity, cause and consequences. Any important protocol modification will be thoroughly communicated with the DSMB members and will obtain amendment clearance from the institutional review board. We do not have a plan to perform interim analyses. Therefore, we do not have stopping guidelines based on the results of interim analyses.

\section{Sample size calculations}

The reported unfavourable clinical outcome rate in patients with severe TBI is $42 \%-59 \% .^{28-30}$ Our data showed that $54 \%$ of patients with TBI undergoing craniotomy for haematoma evacuation had unfavourable outcome. Thus, we suppose the unfavourable clinical outcome rate in the placebo group is $54 \%$, and the anticipated unfavourable clinical outcome rate in the TXA group is reduced by $15 \%(54 \%-46 \%)$. The sample size was calculated with the model of test for two proportions (differences) in PASS V.11 software (NCSS, LLC, USA). Given an alpha level of 0.05 and a beta level of 0.2, the total sample size required is 613 in each group. Considering a $5 \%$ rate of loss to follow-up, it would be necessary 
to include 644 participants per group (total: 1288 participants). There are approximately 40 patients with TBI undergoing craniotomy in Beijing Tian Tan hospital every month. Such a sample size could be achieved in our study.

\section{Data collection and management}

Investigators will explain the benefits of participating in the trial to patients and/or their authorised representative before surgery. Outcome investigators will receive training on all outcome measures. The anaesthesiologist in charge will record the patients' intraoperative data, including blood pressure, heart rate, oxygen saturation, blood loss, urine volume, medicine, infusion volume, transfusion volume and operation time, etc. The data managers will use double data entry to enter data into the EpiData database. An inspector will examine the data, create records and revise these records as necessary. Each participant will have a unique study identifier, and their data will be recorded by an independent data manager. The data will be electronically stored in the EpiData database and undisclosed to other researchers until the study is completed. The final dataset will be handed over to statistical analysts for statistical analysis. Regular data checks and double data entry will be applied to promote data quality.

\section{Record retention and archival}

All the study documents will be archived by the study sites and retained for 3 years after the completion of the study.

\section{Statistical analysis}

Statistical analyses will be performed using Stata V.15.1 statistical software. All measured data will be reported as mean $\pm \mathrm{SD}(\mathrm{x} \pm \mathrm{s}), \mathrm{IQR}(25 \%-75 \%$ percentile $)$ or number $(\%)$.

For the primary outcome, GOSE $1 \sim 4$ will be defined as unfavourable outcomes, GOSE $5 \sim 8$ will be defined as favourable outcomes. $\chi^{2}$ test will be used to evaluate the primary outcome. For the secondary outcomes, we will use t-test or rank-sum test to analyse intraoperative blood loss, blood transfusion volume and CRS-R. $\chi^{2}$ test or Fisher's exact test will be used to analyse the thrombotic event rate and infection rate after surgery. $\chi^{2}$ test or univariate logistic regression analysis will be used for in-hospital and 30-day mortality rate after surgery. Moreover, for the length of hospitalisation and hospitalisation costs, we will use rank-sum test. Subgroup analysis will be performed based on age, sex, GCS score, time from injury to intervention. Interaction analysis might be involved to evaluate the differences between the TXA group and the placebo group in different subgroups.

All randomised participants with informed consent will be analysed. If unintended missing data are related to the primary outcome account for more than $5 \%$, this will be handled with multiple imputation. Analyses will be performed according to the intention-to-treat principle.

\section{Patient and public involvement}

Patients and the public will not be involved in the development of the research or the design of the study. Study results will be disseminated by publication in a medical journal and poster presentation at a medical conference.

\section{DISCUSSION}

This study is a single-centre, randomised, double-blind, placebo-controlled parallel study. We aimed to evaluate the influence of perioperative intravenous TXA treatment on the prognosis of TBI patients undergoing craniotomy for haematoma removal after emergency admission to Beijing Tian Tan Hospital. We also want to explore the influence of TXA on perioperative coagulation function and systemic inflammatory response of patients and its relationship with prognosis.

The WHO has declared TBI to be the leading cause of death and disability worldwide by $2020 .{ }^{31}$ Cerebral haemorrhage is the main clinical manifestation of TBI, and traumatic coagulation disorder is the main cause of progressive aggravation of cerebral haemorrhage. Studies have reported that the incidence of abnormal coagulation function after TBI is $35.2 \%(29.0 \% \sim 41.4 \%)$, and the mortality rate of patients with simple TBI complicated with abnormal coagulation function is $17 \%$ 86\%. ${ }^{32}$ Compared with patients with TBI without coagulation dysfunction, the mortality rate of patients with TBI with coagulation dysfunction is 4.7 times higher, and the risk of poor prognosis is also increased. ${ }^{5}$ The abnormal coagulation function after TBI is directly related to the degree of brain tissue damage. ${ }^{14}$ In addition, systemic inflammatory response after TBI can aggravate coagulation dysfunction in patients with TBI, which is one of the important causes of secondary brain injury. ${ }^{33}$

Studies suggest that the main mechanism leading to abnormal coagulation function after TBI is the activation of tissue plasminogen, hyperfibrinolysis, and disruption of the balance between coagulation and bleeding. ${ }^{34}$ After severe TBI, a strong stress response occurs, which then induces systemic inflammatory response syndrome, leading to increased bleeding, increased blood transfusion and systemic organ failure, aggravating patients' abnormal coagulation function and resulting in increased mortality. ${ }^{35} 36$ In addition, excessive consumption of coagulation factors and platelet dysfunction, ${ }^{37}$ hypoperfusion and protein-C pathway activation, hypothermia, metabolic acidosis and haemodilution can also lead to coagulation dysfunction after TBI. ${ }^{38}$ In patients with TBI, the blood-brain barrier is destroyed; Fbg and its degradation products enter the brain and activate the inflammatory reaction cascade and the immune response in the brain, leading to lymphocyte infiltration, glial cell activation, cytokine release and reactive oxygen element production in the brain. These lead to axonal demyelination and neuronal damage. ${ }^{39}$ Therefore, abnormal coagulation function significantly increases the mortality of patients; the regulation of patients' 
coagulation function has great clinical significance for improving the prognosis of patients, which has attracted more and more attention.

TXA is an antifibrinolytic drug that competitively binds to the fibrinolytic lysine-binding sites and inhibits the fibrinolytic effect of fibrinolytic enzymes. On the other hand, it has anti-inflammatory effects. Plasmin activates and upregulates a cascade of inflammatory responses by activating preinflammatory cells and inducing preinflammatory gene expression. ${ }^{40}$ TXA blocks this inflammatory activation by inhibiting the conversion of plasminogen to plasmin. Patients undergoing cardiopulmonary bypass and orthopaedic joint replacement are generally treated with TXA to reduce systemic inflammation and blood loss. ${ }^{41}$

TXA is widely used in surgical patients who need blood transfusion and haemostatic treatment. It can reduce the risk of bleeding and mortality in patients with TBI. ${ }^{9}$ A large randomised controlled study (CRASH-2) involving 20000 patients in 40 countries found that treatment with TXA within 1 hour after acute TBI reduced the risk of death from bleeding by $30 \%$; treatment with TXA $1-3$ hours after trauma reduced the risk of death by $20 \%$. Therefore, TXA may be a potential treatment for patients with TBI. However, for patients with TBI undergoing craniotomy for haematoma removal, there is still less evidence of the influence of TXA on their prognosis.

Safety is also our important consideration. TXA has been suggested to be at risk of cerebral thrombosis and secondary cerebral ischaemia. ${ }^{42}$ Cerebral ischaemia is a secondary injury after TBI, which can aggravate neurological dysfunction and increase mortality and disability. ${ }^{434}$ TXA may also increase intracranial pressure, leading to intracranial hypoperfusion. ${ }^{45}{ }^{46}$ Thrombotic intravascular coagulation increased the incidence of intracerebral microthrombi. ${ }^{47}$ However, a number of studies have shown that TXA is safe for patients with TBI. ${ }^{6} 1011$ A multicentric, large-sample randomised controlled study (CRASH-3) found that TXA reduced the mortality of patients with mild to moderate TBI without increasing the risk of thrombosis events [risk ratio(RR) $0.98,95 \%$ confidence interval (CI) 0.74 to 1.28], and there was no significant difference in the risk of epilepsy compared with the placebo group (RR $1.09,95 \%$ CI 0.90 to 1.33 ). ${ }^{12}$ Recently, a meta-analysis reported that TXA probably does not increase the risk of AEs. ${ }^{48}$

To sum up, scientific and effective treatments for patients with TBI are needed, and coagulation function is an important clinical indicator affecting the prognosis of patients with TBI. In this study, we aimed to investigate the effect and safety of perioperative intravenous administration of TXA in patients with TBI undergoing craniotomy. We also want to explore the effect of TXA on the inflammatory response and the coagulation function of patients with TBI, as well as its relationship with the prognosis. We aimed to provide a basis for the perioperative TXA treatment for patients with TBI and to optimise the perioperative treatment plan for patients with TBI.

\section{SUMMARY}

In summary, the study aimed to investigate the effect and safety of TXA in patients with TBI undergoing neurosurgery for haematoma removal. If the result of this study is positive, it will provide evidence for optimal perioperative treatment to improve the prognosis of patients with TBI who need craniotomy.

\section{Dissemination}

The results of this study will be disseminated through presentations at scientific conferences and publication in scientific journals.

\section{Audits}

The data monitoring committee will conduct audits through regular interviews, letters or telephone. The data monitoring committee reserves the right to audit the recruitment of patients at any time. The auditing process will be independent from the investigators.

\section{Amendments to the protocol}

Any deviations from the protocol will be fully documented in a report form, reported to all regulatory bodies and thoroughly recorded in a protocol deviation log. The DSMB and PI will determine the protocol amendments. Protocol amendments will be sent as updated protocols to investigators. A copy of each revised protocol will be added to the investigator site file. The protocol will also be updated in the clinical trials registry website.

\section{Strategies to improve the adherence to protocols}

The anaesthesiologist who implements anaesthesia in this study will be trained to obey the standardised procedure. The investigator staff will be well trained to perform preoperative recruitment, assessment and postoperative follow-up. We will train the whole study team to standardly use the assessment scales involves in this study. Moreover, the investigator who will do the assessments will be blind to the intervention.

Contributors BW, WC and YL were involved in the conception and design, data collection and analysis, and manuscript writing. YY, JW, HY and YC were involved in the conception and design, data collection and manuscript revision. All authors read and approved the final manuscript.

Funding This work was supported by the following grants: Beijing Hospital Authority Youth Programme (grant number QML20190508), National Natural Science Foundation of China (grant number 81870865) and Beijing Municipal Administration of Hospitals Incubating Program (grant number PX2019019).

Competing interests None declared.

Patient and public involvement Patients and the public were not involved in the design, conduct, reporting or dissemination plans of this research.

Patient consent for publication Not applicable.

Provenance and peer review Not commissioned; externally peer reviewed.

Open access This is an open access article distributed in accordance with the Creative Commons Attribution Non Commercial (CC BY-NC 4.0) license, which permits others to distribute, remix, adapt, build upon this work non-commercially, and license their derivative works on different terms, provided the original work is properly cited, appropriate credit is given, any changes made indicated, and the use is non-commercial. See: http://creativecommons.org/licenses/by-nc/4.0/. 
ORCID iDs

Yu Lu http://orcid.org/0000-0002-7564-1692

Weihua Cui http://orcid.org/0000-0003-0893-3991

\section{REFERENCES}

1 Talving P, Benfield R, Hadjizacharia P, et al. Coagulopathy in severe traumatic brain injury: a prospective study. J Trauma 2009;66:55-62.

2 Abdelmalik PA, Boorman DW, Tracy J, et al. Acute traumatic coagulopathy accompanying isolated traumatic brain injury is associated with worse long-term functional and cognitive outcomes. Neurocrit Care 2016;24:361-70.

3 Frontera JA, Lewin JJ, Rabinstein AA, et al. Guideline for reversal of antithrombotics in intracranial hemorrhage: a statement for healthcare professionals from the neurocritical care Society and society of critical care medicine. Neurocrit Care 2016;24:6-46.

4 de Oliveira Manoel AL, Neto AC, Veigas PV, et al. Traumatic brain injury associated coagulopathy. Neurocrit Care 2015;22:34-44.

5 Albert V, Arulselvi S, Agrawal D, et al. Early posttraumatic changes in coagulation and fibrinolysis systems in isolated severe traumatic brain injury patients and its influence on immediate outcome. Hematol Oncol Stem Cell Ther 2019;12:32-43.

6 Ker K, Roberts I, Shakur H. Antifibrinolytic drugs for acute traumatic injury. Cochrane Database Syst Rev 2015;5:CD004896.

7 CRASH-2 collaborators, Roberts I, Shakur $\mathrm{H}$, et al. The importance of early treatment with tranexamic acid in bleeding trauma patients: an exploratory analysis of the $\mathrm{CRASH}-2$ randomised controlled trial. Lancet 2011;377:e1-2.

8 CRASH-2 trial collaborators, Shakur H, Roberts I, et al. Effects of tranexamic acid on death, vascular occlusive events, and blood transfusion in trauma patients with significant haemorrhage (CRASH-2): a randomised, placebo-controlled trial. Lancet 2010;376:23-32.

9 Yokobori S, Yatabe T, Kondo Y, et al. Efficacy and safety of tranexamic acid administration in traumatic brain injury patients: a systematic review and meta-analysis. J Intensive Care 2020;8:46.

10 Perel P, Al-Shahi Salman R, Kawahara T, et al. CRASH-2 (Clinical Randomisation of an Antifibrinolytic in Significant Haemorrhage) intracranial bleeding study: the effect of tranexamic acid in traumatic brain injury--a nested randomised, placebo-controlled trial. Health Technol Assess 2012;16:1-54

11 Yutthakasemsunt S, Kittiwatanagul W, Piyavechvirat P, et al. Tranexamic acid for patients with traumatic brain injury: a randomized, double-blinded, placebo-controlled trial. BMC Emerg Med 2013;13:20.

12 CRASH-3 trial collaborators. Effects of tranexamic acid on death, disability, vascular occlusive events and other morbidities in patients with acute traumatic brain injury (CRASH-3): a randomised, placebocontrolled trial. Lancet 2019;394:1713-23.

13 Maegele M, Schöchl H, Menovsky T, et al. Coagulopathy and haemorrhagic progression in traumatic brain injury: advances in mechanisms, diagnosis, and management. Lancet Neurol 2017;16:630-47.

14 Kearney TJ, Bentt L, Grode M, et al. Coagulopathy and catecholamines in severe head injury. J Trauma 1992;32:608-12.

15 Carney N, Totten AM, O'Reilly C, et al. Guidelines for the management of severe traumatic brain injury, fourth edition. Neurosurgery 2017;80:6-15.

16 Abdelmalik PA, Draghic N, Ling GSF. Management of moderate and severe traumatic brain injury. Transfusion 2019;59:1529-38.

17 Capizzi A, Woo J, Verduzco-Gutierrez M. Traumatic brain injury: an overview of epidemiology, pathophysiology, and medical management. Med Clin North Am 2020;104:213-38.

18 Seule M, Brunner T, Mack A, et al. Neurosurgical and intensive care management of traumatic brain injury. Facial Plast Surg 2015;31:325-31.

19 Galgano M, Toshkezi G, Qiu X, et al. Traumatic brain injury: current treatment strategies and future Endeavors. Cell Transplant 2017;26:1118-30.

20 Yeatts SD, Martin RH, Meurer W, et al. Sliding scoring of the Glasgow outcome Scale-Extended as primary outcome in traumatic brain injury trials. J Neurotrauma 2020;37:2674-9.

21 Nichol AD, Higgins AM, Gabbe BJ, et al. Measuring functional and quality of life outcomes following major head injury: common scales and checklists. Injury 2011;42:281-7.

22 Bagiella E, Novack TA, Ansel B, et al. Measuring outcome in traumatic brain injury treatment trials: recommendations from the traumatic brain injury clinical trials network. J Head Trauma Rehabil 2010;25:375-82.
23 Ardolino A, Sleat G, Willett K. Outcome measurements in major trauma--results of a consensus meeting. Injury 2012;43:1662-6.

24 McMillan T, Wilson L, Ponsford J, et al. The Glasgow Outcome Scale -40 years of application and refinement. Nat Rev Neurol 2016;12:477-85.

25 Jaramillo S, Montane-Muntane M, Capitan D, et al. Agreement of surgical blood loss estimation methods. Transfusion 2019;59:508-15.

26 Sattin D, Minati L, Rossi D, et al. The coma recovery scale modified score: a new scoring system for the coma recovery Scale-revised for assessment of patients with disorders of consciousness. Int $J$ Rehabil Res 2015;38:350-6.

27 Bodien YG, Carlowicz CA, Chatelle C, et al. Sensitivity and Specificity of the Coma Recovery Scale--Revised Total Score in Detection of Conscious Awareness. Arch Phys Med Rehabil 2016;97:490-2.

28 Barthélemy EJ, Melis M, Gordon E, et al. Decompressive craniectomy for severe traumatic brain injury: a systematic review. World Neurosurg 2016;88:411-20.

29 Bonow RH, Barber J, Temkin NR, et al. The outcome of severe traumatic brain injury in Latin America. World Neurosurg 2018;111:e82-90.

30 Cooper DJ, Rosenfeld JV, Murray L, et al. Patient outcomes at twelve months after early decompressive craniectomy for diffuse traumatic brain injury in the randomized DECRA clinical trial. J Neurotrauma 2020;37:810-6.

31 Hyder AA, Wunderlich CA, Puvanachandra P, et al. The impact of traumatic brain injuries: a global perspective. NeuroRehabilitation 2007;22:341-53.

32 Epstein DS, Mitra B, O'Reilly G, et al. Acute traumatic coagulopathy in the setting of isolated traumatic brain injury: a systematic review and meta-analysis. Injury 2014;45:819-24.

33 Brain Trauma Foundation, American Association of Neurological Surgeons, Congress of Neurological Surgeons, et al. Guidelines for the management of severe traumatic brain injury. IX. cerebral perfusion thresholds. J Neurotrauma 2007;24 Suppl 1:S59-64.

34 Nakae R, Takayama Y, Kuwamoto K, et al. Time course of coagulation and fibrinolytic parameters in patients with traumatic brain injury. J Neurotrauma 2016;33:688-95.

35 Wada T, Gando S, Maekaw K, et al. Disseminated intravascular coagulation with increased fibrinolysis during the early phase of isolated traumatic brain injury. Crit Care 2017;21:219.

36 Walker PF, Foster AD, Rothberg PA, et al. Tranexamic acid decreases rodent hemorrhagic shock-induced inflammation with mixed endorgan effects. PLoS One 2018;13:e0208249.

37 Solomon C, Traintinger S, Ziegler B, et al. Platelet function following trauma. A multiple electrode aggregometry study. Thromb Haemost 2011;106:322-30.

38 Albert V, Subramanian A, Agrawal D, et al. Acute traumatic Endotheliopathy in isolated severe brain injury and its impact on clinical outcome. Med Sci 2018;6. doi:10.3390/medsci6010005. [Epub ahead of print: 16 Jan 2018]

39 Ziliotto N, Bernardi F, Jakimovski D, et al. Coagulation pathways in neurological diseases: multiple sclerosis. Front Neurol 2019;10:409.

40 Singleton $\mathrm{Q}$, Vaibhav K, Braun M, et al. Bone marrow derived extracellular vesicles activate osteoclast differentiation in traumatic brain injury induced bone loss. Cells 2019;8. doi:10.3390/ cells8010063. [Epub ahead of print: 1701 2019].

41 Wang D, Yang Y, He C, et al. Effect of multiple doses of oral tranexamic acid on haemostasis and inflammatory reaction in tota hip arthroplasty: a randomized controlled trial. Thromb Haemost 2019;119:092-103.

42 Gando S, Sawamura A, Hayakawa M, Trauma HM. Trauma, shock, and disseminated intravascular coagulation: lessons from the classical literature. Ann Surg 2011;254:10-19.

43 Mazzeo AT, Kunene NK, Choi S, et al. Quantitation of ischemic events after severe traumatic brain injury in humans: a simple scoring system. J Neurosurg Anesthesiol 2006;18:170-8.

44 Hulka F, Mullins RJ, Frank EH. Blunt brain injury activates the coagulation process. Arch Surg 1996;131:923-7.

45 Burke JF, Stulc JL, Skolarus LE, et al. Traumatic brain injury may be an independent risk factor for stroke. Neurology 2013;81:33-9.

46 Baharoglu MI, Germans MR, Rinkel GJE, et al. Antifibrinolytic therapy for aneurysmal subarachnoid haemorrhage. Cochrane Database Syst Rev 2013;8:CD001245.

47 Kaufman $\mathrm{HH}$, Hui KS, Mattson JC, et al. Clinicopathological correlations of disseminated intravascular coagulation in patients with head injury. Neurosurgery 1984;15:34-42.

48 Lawati KA, Sharif S, Maqbali SA, et al. Efficacy and safety of tranexamic acid in acute traumatic brain injury: a systematic review and meta-analysis of randomized-controlled trials. Intensive Care Med 2021;47:14-27. 